\title{
Liver Undifferentiated Carcinoma
}

National Cancer Institute

\section{Source}

National Cancer Institute. Liver Undifferentiated Carcinoma. NCI Thesaurus. Code

C96792.

A rare carcinoma that arises from the liver. The diagnosis is made by

immunohistochemical studies. Morphologic studies alone cannot establish the diagnosis

of carcinoma or further subclassify the malignant tumor. 